\title{
Ipsilateral hamstring tendon graft reconstruction for chronic patellar tendon ruptures: surgical technique
}

\author{
Nicola Maffulli ${ }^{1}$ \\ Angelo Del Buono ${ }^{2}$ \\ Francesco Oliva ${ }^{3}$ \\ 1 Department of Musculoskeletal Disorders, Faculty \\ of Medicine and Surgery, University of Salerno, \\ Salerno, Italy; Centre for Sports and Exercise \\ Medicine, Mile End Hospital, Barts and The London \\ School of Medicine and Dentistry, London, UK \\ 2 Department of Orthopedic and Trauma Surgery, \\ Fidenza Hospital, Fidenza, Italy \\ 3 Department of Orthopaedics and Traumatology, \\ University of Rome "Tor Vergata", Rome, Italy
}

Corresponding author:

Nicola Maffulli

Department of Musculoskeletal Disorders, University of Salerno

Via Salvatore Allende

84081 Baronissi, Salerno, Italy

E-mail: n.maffulli@qmul.ac.uk

\section{Summary}

Background: Patellar tendon chronic ruptures are more debated to manage that acute injuries as the patella tends to retract proximally (after 2 weeks), and surrounding soft tissues may make the repair more complicate.

Purpose: We propose a novel technique in which the patellar tendon is reconstructed using ipsilateral hamstring tendons. This surgical procedure implies to drill a single tunnel through the patella and another through the tibia to reduce the risk of bony breakage. In addition, the tendon is secured to the bone, at the tunnel exit points, by periostium sutures without requiring any additional surgery for hardware removal.

Methods: We assessed 19 patients (16 men and 3 women) who underwent $\mathrm{PT}$ reconstruction at an average of 5.8 years (range, 4 to 7.8 years, SD: 3.5) from the index surgery. The mean age at surgery was $46 \pm 9.2$ years (range, $38-59$ years).

Results: All patients had a complete tear of the patellar tendon.

Conclusions: The main indication for this procedure is the reconstruction of the PT in patients with chronic rupture ( $>6$ weeks) in which the ten- don gap is greater than 2 centimeters and the scar tissues and degenerated tendon ends do not allow to juxtapose the tendon stumps to each other. Level of evidence: Level IV.

KEY WORDS: patellar tendon, tendon repair, patellar tendon ruptures, chronic patellar tendon ruptures, patellar tendon repair.

\section{Introduction}

Autologous ipsilateral hamstring tendon graft reconstruction for management of chronic patellar tendon ruptures is strong, respects the native tissues, and allows early mobilization and a high rate of return to pre-injury activities.

Chronic ruptures are more debated to manage that acute injuries as the patella tends to retract proximally (after 2 weeks), and surrounding soft tissues may make the repair more complicate ${ }^{1,2}$. In these instances, tendon augmentations strengthen the construct, and allow earlier return to motion. Specifically, autologous semitendinosus and gracilis tendon grafts ${ }^{3,4}$, contralateral bone-patellar tendon-bone ${ }^{5}$, turndown of the quadriceps tendon ${ }^{6}$, lateral gastrocnemius muscle belly and part of an Achilles tendon ${ }^{7}$, extensor-mechanism allograft using bone-patellar tendon-bone allograft ${ }^{8}$ and Achilles tendon ${ }^{9,10}$, and artificial materials ${ }^{11}$ have been used. However, the best management of these lesions is still controversial. We propose a novel technique in which the patellar tendon is reconstructed using ipsilateral hamstring tendons. Differently from other procedures in which 2 tunnels are made to the patella and tibial tuberosity, this technique implies to drill a single tunnel through the patella and another through the tibia to reduce the risk of bony breakage. In addition, the tendon is secured to the bone, at the tunnel exit points, by periostium sutures without requiring any additional surgery for hardware removal. In our experience, this procedure has a low rate of complications and provides results that are at least comparable with those reported with other surgical approaches. The technique consists of seven steps.

Step 1: Position

Step 2: Incision

Step 3: Harvest visualization

Step 4: Harvest preparation

Step 5: Tunnel drilling

Step 6: Tunnel passage

Step 7: Graft fixation and closure. 


\section{Surgical technique}

\section{Step 1: Position}

Put the patient in supine position

- Under general anaesthesia, position the patient supine, prep and drape the knee in the usual sterile fashion.

- Flex the knee to $90^{\circ}$.

\section{Step 2: Incision}

Make a mid-line longitudinal incision from the proximal pole of the patella to approximately $3 \mathrm{~cm}$ distal to the tibial tuberosity.

- Make a mid-line incision from the proximal pole of the patella to approximately $3 \mathrm{~cm}$ distal to the tibial tuberosity.

- Expose the patellar tendon ends and free them from surrounding fibrotic adhesions and scar tissue (Fig. 1).

\section{Step 3: Harvest visualization}

Visualize and prepare the tendons.

- Through the same incision, dissect the fascia and surrounding soft tissues to identify the pes anserinus.

- Cut the fascia and grab using a mosquito. The tendons are often adherent because of the presence of adhesions.

- Separate the tendons: the semitendinosus tendon is lower, thicker and bigger; the gracilis is above the semitendinosus, thinner.

\section{Step 4: Harvest preparation}

Free the tendons of gracilis and semitendinosus of surrounding tissues and vinculae, and pass them through an open tendon stripper.
- Harvest the proximal edge of both tendons.

- Prepare the proximal ends in the usual fashion using five continuous 2 sided Number 1 Vicryl (Ethicon, Edinburgh, Scotland) whip stitches (to tubolarise the tendon edges) (Fig. 2).

- Take care to leave the distal tendon ends attached in situ, attached to the tibia, and suture them in the same way.

\section{Step 5: Tunnel drilling}

Drill a transverse tunnel through the mid portion of the patella and another transverse tunnel at least 2 $\mathrm{cm}$ posterior to the tibial tuberosity.

- With the knee extended, after mobilization and exposure of the distal half of the patella, drill a transverse tunnel through the mid portion of the patella with a cannulated burr over a Kirschner wire (Fig. 3).

- Then, drill another transverse tunnel at least $2 \mathrm{~cm}$ posterior to the tibial tuberosity. Drill both tunnels lateral to medial. To improve the stability of the construct, reattach the proximal and distal ends of the tendon medially and laterally in a Krachow suture.

- The 2 tunnels are of equal diameter, usually 7 $\mathrm{mm}$. Insert a guide wire and a shuttle suture into the hole to pass the tendon graft through the patellar tunnel from lateral to medial (Fig. 4).

\section{Step 6: Tendon passage}

Pass the tendon into the bone

- Pass the tendon through the patellar tunnel from medial to lateral.

- Cross over the tendon ends in a figure of eight fashion.

- Switch the ends through the tibial tunnel (Fig. 5).

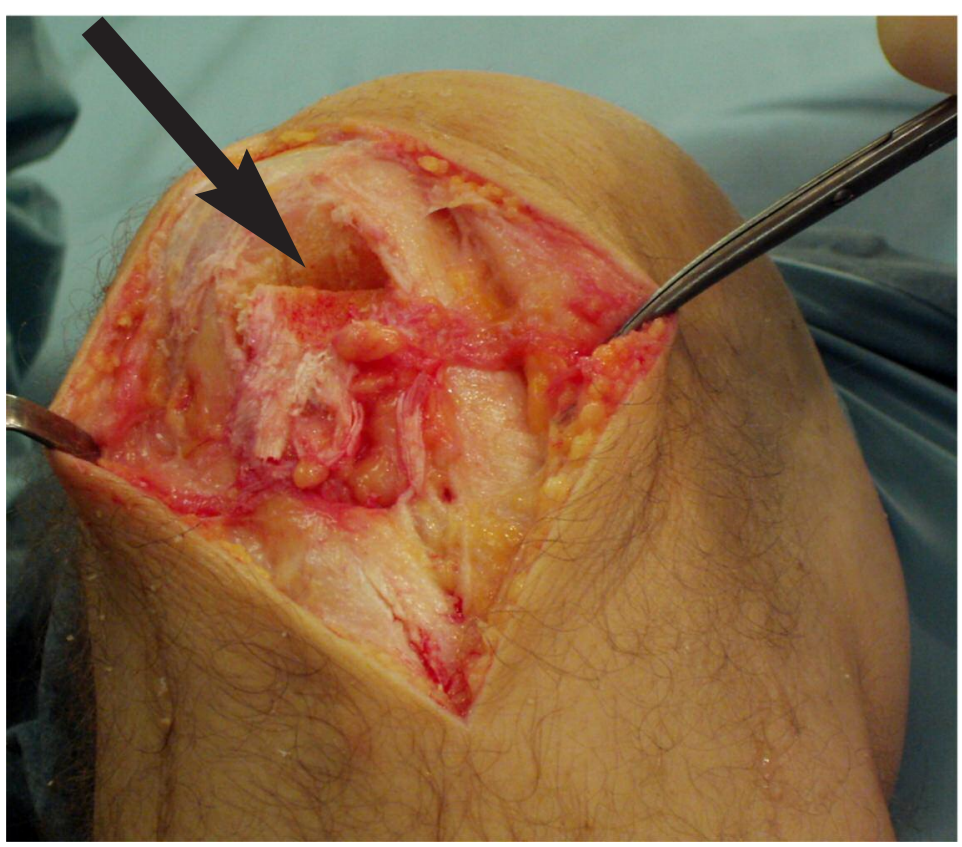

Figure 1. With the patient in the supine position, a mid-line incision is made from the proximal pole of the patella to approximately $3 \mathrm{~cm}$ distal to the tibial tuberosity. The patellar tendon ends are exposed, and released from surrounding adherences and scar tissues. The arrow indicates the upper end of a disrupted patellar still attached to the lower pole of the patella. 

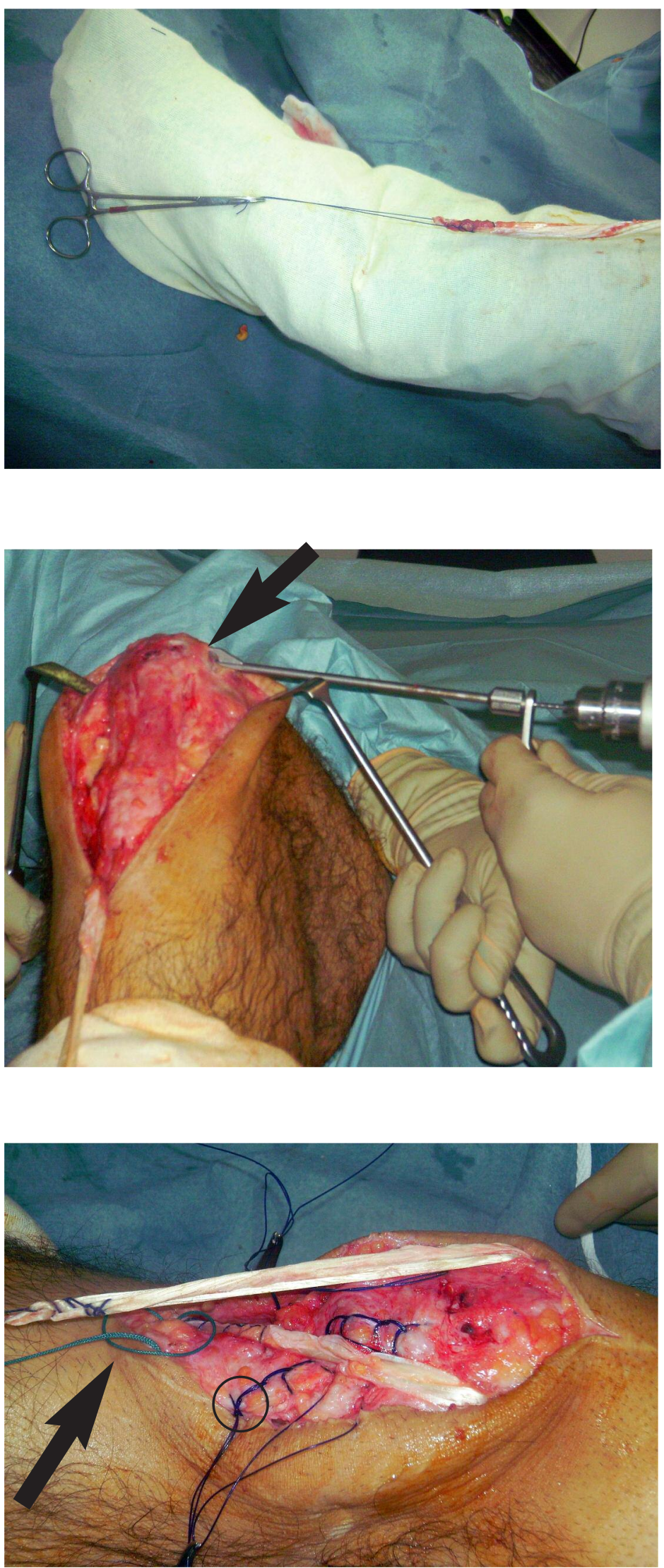

Figure 2. The proximal ends of the tendons are prepared using five continuous 2 sided No. 1 Vicryl (Ethicon, Edinburgh, Scotland) whip stitches.

Figure 3. The knee is flexed to $90^{\circ}$. A tunnel is drilled transversely, lateral to medial, in the mid portion of the patella, paying attention not to damage the articular surface of the patellar bone. The black arrow indicates the lateral entry point through which the canulated burr and Kirschner wire are inserted.

Figure 4. The free edges of both the tendons are passes through the tunnel made into the patella. A fiber wire working as shuttle is used to pass the graft through the hole from lateral to medial into the patella. The arrow shows the passage of the graft behind the tibial tuberosity. The black circle shows the krachow suture for repairing the tendon. 


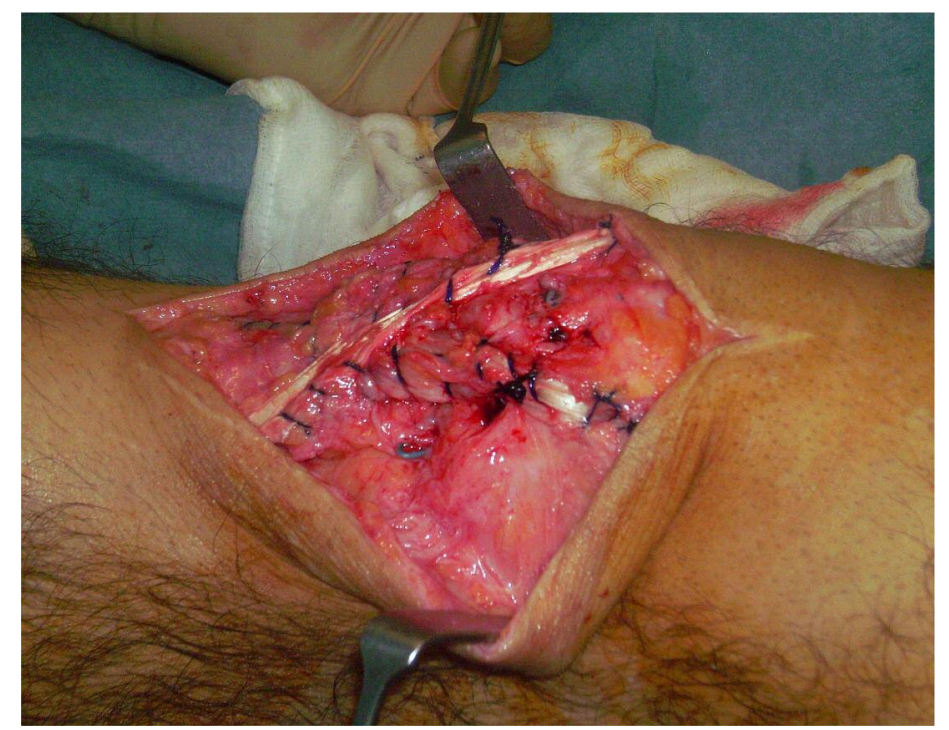

Figure 5. Definitive image of the tendons, crossed over in a figure of eight configuration.

\section{Step 7: Graft fixation and closure}

Secure the graft to the patella tunnel exit holes with periosteum sutures and to the distal tunnel using a bio-absorbable interference screw.

- Apply traction to the patella to try and relocate it as close as possible to its physiological position, without attempting to release the quadriceps tendon or further dissect the peri-patellar tissues.

- Secure the graft to the patella tunnel exit holes with absorbable tendon to periosteum sutures, and in the distal tunnel using a bio-absorbable 7 $\mathrm{mm}$ diameter interference screw.

- Juxtapose the subcutaneous fat using fine absorbable sutures, close the skin with subcuticular absorbable sutures.

- The leg is immobilized in full extension using a cylinder cast leaving the ankle free.

\section{Results}

We assessed 19 patients (16 men and 3 women) who underwent $\mathrm{PT}$ reconstruction at an average of 5.8 years (range, 4 to 7.8 years, SD: 3.5 ) from the index surgery ${ }^{12,13}$. The mean age at surgery was $46 \pm 9.2$ years (range, 38-59 years). Six patients had undergone previous ipsilateral patellar tendon repair, and had re-ruptured their patellar tendon within six months of the original rupture; no patient had received previous reconstruction. The 13 patients who presented with a primary chronic rupture had sustained the index injury $3.8 \pm 2.1$ months (range 8 weeks to 7 months) before the procedure. All patients had a complete tear of the patellar tendon. In the six patients in whom a previous repair had been performed, the re-tear occurred in the mid-substance of the tendon in 4 patients, through the sutures, and at the attachment of the tendon on the lower pole of the patella in the remaining two.
None of the patients had a clinically significant loss of knee flexion at the last follow-up. The mean preoperative knee flexion of $110^{\circ}$ (range, $90^{\circ}$ to $125^{\circ}$ ) substantially increased postoperatively to $132^{\circ}$ (range, $115^{\circ}$ to $135^{\circ}$ ). The mean preoperative extension lag of $20^{\circ}$ substantially decreased to $3^{\circ}$ postoperatively, with 15 of 19 patients showing no evidence of extensor lag at the last follow-up.

The mean modified Cincinnati score increased from $44.5 \pm 7.6$ (range, 33-61) at baseline to $84 \pm 5.7$ (range, 73-91; $\mathrm{P}<.0001$ ) postoperatively; the mean preoperative Kujala score of $42 \pm 6.8$ (range, 30-55) increased to $81 \pm 6.1$ (range, 69-91; $\mathrm{P}<.0001$ ) at the last follow-up. At the last follow-up, 9 patients had degeneration of the patellofemoral joint, but their scores were not significantly different compared to patients without any changes. The mean preoperative InsallSalvati index of 1.8 (range, 1.4-2.1) decreased to an average index of 1.5 (range, 1.2-1.8; $\mathrm{P}=<.0001$ ) at the latest follow-up.

All patients had returned to ordinary daily and working activities. Specifically, 11 patients were manual workers: all returned to manual working activities; 8 were sedentary workers: all returned to sedentary working activities. At the last follow, of 8 patients who practiced sport preoperatively, all at recreational level, 5 had returned to the same activity level. Three patients had abandoned any sport activity; 1 patient had stopped because of persistent pain to the knee and 2 patients felt uncomfortable with their knee. Fourteen patients $(73.7 \%)$ were very satisfied with the procedure, $3(15.8 \%)$ satisfied, 1 moderately satisfied, and 1 unsatisfied. To assess the relationship between occurrence of degenerative changes and age at surgery with postoperative satisfaction, we grouped the patients according to the age at surgery ( $<45$ or $>45$ years old) and occurrence of degenerative changes (presence or absence). We found that neither the age at surgery $(\mathrm{P}=0.93)$ nor the develop- 
ment of degenerative changes $(P=0.11)$ affected the status of satisfaction.

At the last follow-up, the muscle volume of the thigh on the operated side was significantly greater $(5.3 \pm$ $1.4 \mathrm{~L})$ than at baseline $(4.8 \pm 1.1)(\mathrm{P}=0.02)$, but it remained lower than the non-injured contra-lateral limb $(5.7 \pm 1.3 \mathrm{~L}$ preoperatively, and $5.8 \pm 1.4 \mathrm{~L}$ at followup, $\mathrm{P}=.04)$. The maximal voluntary isometric strength assessment showed that the operated limb remained significantly weaker than the contralateral $\operatorname{leg}(521 \pm 152.6$ vs $713 \pm 125.5 \mathrm{~N} ; \mathrm{P}=0.01)$.

\section{Complications}

When performing the transverse drill hole, the anterior edge of the lateral aspect of the patella was partially breached in one patient: the periosteum of the anterior aspect of the patella was sutured to the tendon with absorbable stitches after passage of the graft. In another patient, the anterior aspect of the tibial tuberosity was partially detached and, in the same way, the tendon ends of the graft were sutured close to the bone. These patients were advised to wear a brace locked in extension for 4 weeks, and healed without any sequelae. Five patients reported hypoesthesia and numbness over the anterior aspect of the knee, but one patient still reported an area of hypoesthesia over the anterior tibial tuberosity at the final follow-up, with no inconvenience. Three patients reported persistent anterior knee pain: all of them had evidence of grade III degenerative changes to the patella at the index procedure. We did not experience any patellar re-rupture, patellar fracture, vascular complication, or infection.

\section{What to watch for}

\section{Indications}

The main indication for this procedure is the reconstruction of the PT in patients with chronic rupture ( $>6$ weeks) in which the tendon gap is greater than 2 centimeters and the scar tissues and degenerated tendon ends do not allow to juxtapose the tendon stumps to each other.

\section{Contraindications}

- Patellar disorders;

- Previous ipsilateral hamstring tendon graft anterior cruciate ligament reconstruction;

- Impossibility to undertake appropriate post operative care.

\section{Pitfalls \& Challenges}

- The incision should be extended from the proximal pole of the patella to approximately $3 \mathrm{~cm}$ distal to the tibial tuberosity to provide wide dissection and exposure of pes anserinus;

- The hamstring tendons are not detached from the tibial insertion site because when passed in oppo- site directions through the tunnels, force is distributed on both sides of the original patellar tendon, allowing to restore the correct patellar location for maximal range of motion of the knee joint.

- Drilling a single tunnel into the patella and tibial tuberosity without any cerclage wiring supplementation lowers the risks for bony fractures and, as potential advantage of the technique, requires no hardware removal and prevents limited range of motion.

- The tendon is sutured to the bony entry and exit points of both the tibial and femoral tunnels, and an interference screw is inserted into the tunnel through the tibial tubercle to improve the stability of the construct.

- The rehabilitation program should be extensive, and steadily supervised by appropriately trained health care professionals throughout the process.

- Plaster immobilization is indicated in the period immediately after the operation to prevent excessive stresses to the knee joint and keep the position of both the patella and patellar tendon. In this way, the healing process would be stimulated, and also accelerated.

- The time period of plaster immobilization is 2 weeks to prevent occurrence of stiffness and anterior knee pain, both causes over time of surgical failure.

\section{Clinical comments}

\section{Complications}

Compared to other procedures, the hamstring tendons contain stronger fibers than the distal iliotibial tract, fascia lata, or quadriceps-patellar retinaculum, and ensure a strong integration to the tendon bone interface. We believe this techniques better restores the range of motion and lower the risks of iatrogenic bone fractures to the patella and tibial tubercle.

\section{Suggestions}

We propose this procedure as it does not require any additional surgery for hardware removal.

\section{Ethics approval}

All procedures described in this study were approved by the Ethics Committee of our Institution. All patients gave their informed consent in written form. Furthermore the manuscript follow the ethical to international standards according and as required by the MLTJ Journal. ${ }^{14}$

\section{Conflict of interest}

The Author has no financial or personal relationships with other people or organizations that could inappropriately influence their work. 


\section{References}

1. Enad JG. Patellar tendon ruptures. South Med J. 1999;92: 563-566.

2. Chen B, Li R, Zhang S. Reconstruction and restoration of neglected ruptured patellar tendon using semitendinosus and gracilis tendons with preserved distal insertions: two case reports. Knee. 2012;19:508-512.

3. Cadambi A, Engh GA. Use of a semitendinosus tendon autogenous graft for rupture of the patellar ligament after total knee arthroplasty. A report of seven cases. J Bone Joint Surg Am. 1992;74:974-979.

4. Bek D, Demiralp B, Komurcu M, Sehirlioglu A. Neglected patellar tendon rupture: a case of reconstruction without quadriceps lengthening. J Orthop Traumatol. 2008;9:39-42.

5. Milankov MZ, Miljkovic N, Stankovic M. Reconstruction of chronic patellar tendon rupture with contralateral BTB autograft: a case report. Knee Surg Sports Traumatol Arthrosc. 2007;15:1445-1448

6. Scuderi C. Ruptures of the quadriceps tendon; study of twenty tendon ruptures. Am J Surg. 1958;95:626-634.

7. Chiou HM, Chang MC, Lo WH. One-stage reconstruction of skin defect and patellar tendon rupture after total knee arthroplasty. A new technique. J Arthroplasty. 1997;12:575-579.
8. ElGuindy A, Lustig S, Servien E, et al. Treatment of chronic disruption of the patellar tendon in Osteogenesis Imperfecta with allograft reconstruction. Knee. 2011;18:121-124.

9. Crossett LS, Sinha RK, Sechriest VF, Rubash HE. Reconstruction of a ruptured patellar tendon with achilles tendon allograft following total knee arthroplasty. J Bone Joint Surg Am. 2002;84-A:1354-1361.

10. Lewis PB, Rue JP, Bach BR Jr. Chronic patellar tendon rupture: surgical reconstruction technique using 2 Achilles tendon allografts. J Knee Surg. 2008;21:130-135.

11. Fukuta S, Kuge A, Nakamura M. Use of the Leeds-Keio prosthetic ligament for repair of patellar tendon rupture after total knee arthroplasty. Knee. 2003;10:127-130.

12. Maffulli N, Del Buono A, Loppini M, Denaro V. Ipsilateral hamstring tendon graft reconstruction for chronic patellar tendon ruptures: average 5.8 year follow up retrospective study. $J$ Bone Joint Surg Am. In Press.

13. Spoliti M, Giai Via A, Padulo J, Oliva F, Del Buono A, Maffulli N Surgical repair of chronic patellar tendon rupture in total knee replacement with ipsilateral hamstring tendons. Knee Surg Sports Traumatol Arthrosc. 2014.

14. Padulo J, Oliva F, Frizziero A, Maffulli N. Muscles, Ligaments and Tendons Journal - Basic principles and recommendations in clinical and field science research: 2016 Update. MLTJ. 2016;6(1):1-5. 